\title{
Advanced Medical Use of Three-Dimensional Imaging in Congenital Heart Disease: Augmented Reality, Mixed Reality, Virtual Reality, and Three-Dimensional Printing
}

\author{
Hyun Woo Goo, MD, PhD ${ }^{1}$, Sang Joon Park, PhD ${ }^{2}$, Shi-Joon Yoo, MD $^{3}$ \\ ${ }^{1}$ Department of Radiology and Research Institute of Radiology, University of Ulsan College of Medicine, Asan Medical Center, Seoul, Korea; \\ ${ }^{2}$ Department of Radiology, Biomedical Research Institute, Seoul National University Hospital, Seoul, Korea; ${ }^{3}$ Department of Diagnostic Imaging, \\ The Hospital for Sick Children, University of Toronto, Toronto, Canada
}

Three-dimensional (3D) imaging and image reconstruction play a prominent role in the diagnosis, treatment planning, and post-therapeutic monitoring of patients with congenital heart disease. More interactive and realistic medical experiences take advantage of advanced visualization techniques like augmented, mixed, and virtual reality. Further, 3D printing is now used in medicine. All these technologies improve the understanding of the complex morphologies of congenital heart disease. In this review article, we describe the technical advantages and disadvantages of various advanced visualization techniques and their medical applications in the field of congenital heart disease. In addition, unresolved issues and future perspectives of these evolving techniques are described.

Keywords: Augmented reality; Congenital heart disease; 3D imaging; 3D modeling; 3D printing; Virtual reality

\section{INTRODUCTION}

Recently improved three-dimensional (3D) cardiac imaging technologies are triggering the medical use of advanced visualization techniques including augmented reality, mixed reality, virtual reality, and 3D printing. The driving factor is the vast number of 3D cardiac images, acquired from patients with congenital heart disease (CHD), serving as a source of data for these new visualization techniques. Non-invasive visualization of accurate patient-specific cardiovascular anatomy is essential for the diagnosis and treatment of CHD, not only for accessing morphological complexity but also due to the difficulty deciding among the available treatment

Received August 20, 2019; accepted after revision October 24, 2019. Corresponding author: Hyun Woo Goo, MD, PhD, Department of Radiology and Research Institute of Radiology, University of Ulsan College of Medicine, Asan Medical Center, 88 Olympic-ro 43-gil, Songpa-gu, Seoul 05505, Korea.

- Tel: (822) 3010-4388 - Fax: (822) 476-0090

- E-mail: ghw68@hanmail.net

This is an 0pen Access article distributed under the terms of the Creative Commons Attribution Non-Commercial License (https://creativecommons.org/licenses/by-nc/4.0) which permits unrestricted non-commercial use, distribution, and reproduction in any medium, provided the original work is properly cited. options. Several review articles describing the techniques and clinical applications of augmented reality, mixed reality, virtual reality, and 3D printing have been published (1-4). However, these articles tend to focus on one technology. In this regard, it is necessary for a comprehensive review that provides a balanced view and integrative comparisons among these advanced visualization techniques.

Further, highly advanced medical 3D workstations (extensively used in radiology) are underutilized as tools to support these new technologies. Therefore, the usefulness of these workstations for cardiac computed tomography (CT) and magnetic resonance imaging (MRI), for evaluating CHD, will be explored in this article. Finally, we describe technical considerations and medical applications of augmented reality, mixed reality, virtual reality, and 3D printing, including unresolved issues and future perspectives.

\section{Three-Dimensional Imaging in Congenital Heart Disease}

CT and MRI are used for 3D visualization of cardiovascular structures in CHD. In contrast, 3D echocardiography is limited by operator skill and an incomplete acoustic window. In this 
section, we focus on 3D imaging using cardiac CT and MRI.

\section{Data Acquisition}

Ideal 3D images for CHD have an isotropic high spatial resolution, sharp contrast between blood and myocardium, homogeneous signal distribution across all cardiovascular structures, low image noise, and minimal artifacts. Therefore, obtaining all these features is the gold standard for 3D data acquisition in CHD. These features make all post-processing steps and advanced visualization techniques described in the following sections more accurate and less time-consuming.

CT is increasingly used for evaluating CHD because the imaging modality is exceedingly fast and can provide excellent image quality $(5,6)$. In addition, the $\mathrm{CT}$ radiation dose can be minimized by combining dose reduction strategies while maintaining image quality $(7,8)$. Through electrocardiography (ECG)-synchronized CT scanning, crisp cardiovascular images can be obtained without meaningful cardiac pulsation artifacts. Further, iterative reconstruction algorithms can be used to reduce image noise and may eliminate the need for additional smoothing processes. Moreover, model-based iterative reconstruction algorithms may increase image sharpness. Optimization of an intravenous injection protocol is crucial for achieving uniform cardiovascular enhancement without substantial perivenous streak artifacts. A user-friendly, vendor-specific pediatric cardiothoracic CT guideline was recently published and can be utilized to optimize the CT protocols of clinicians and institutions (9).

MRI does not use ionizing radiation, but comprehensive examination is limited by the lengthy scanning times. A detailed cardiovascular MRI guideline for CHD was published in 2013 (10). 'Non-ECG-synchronized contrast-enhanced magnetic resonance angiography' and 'ECG-triggered navigator-gated T2-prepared balanced steady-state free precession imaging' are two 3D MRI methods with almost isotropic spatial resolution used for evaluating CHD. However, the accurate evaluation of intracardiac structures, coronary arteries, and proximal great arteries is noticeably impeded by cardiac pulsation artifacts in the former, and the latter is also suboptimal in image quality due to different signal intensities, lower spatial resolution, and more artifacts (Fig. 1). Intravenous administration of gadofosveset trisodium (11) and ferumoxytol (12) was used to improve the image quality of cardiac MRI. However, these two MRI contrast agents were recently withdrawn due to poor sales and potential safety issues.

\section{Post-Processing}

A variety of useful volumetric segmentation tools, such as threshold-based segmentation and 3D region-growing algorithms, are available for post-processing the 3D cardiac images of CHD. Data in 3D are often presented as a single set of volume-rendered images. Multiple sets of different volumes may be color-coded, and spatial relationships with adjacent structures, including the airways and the thoracic cage, can
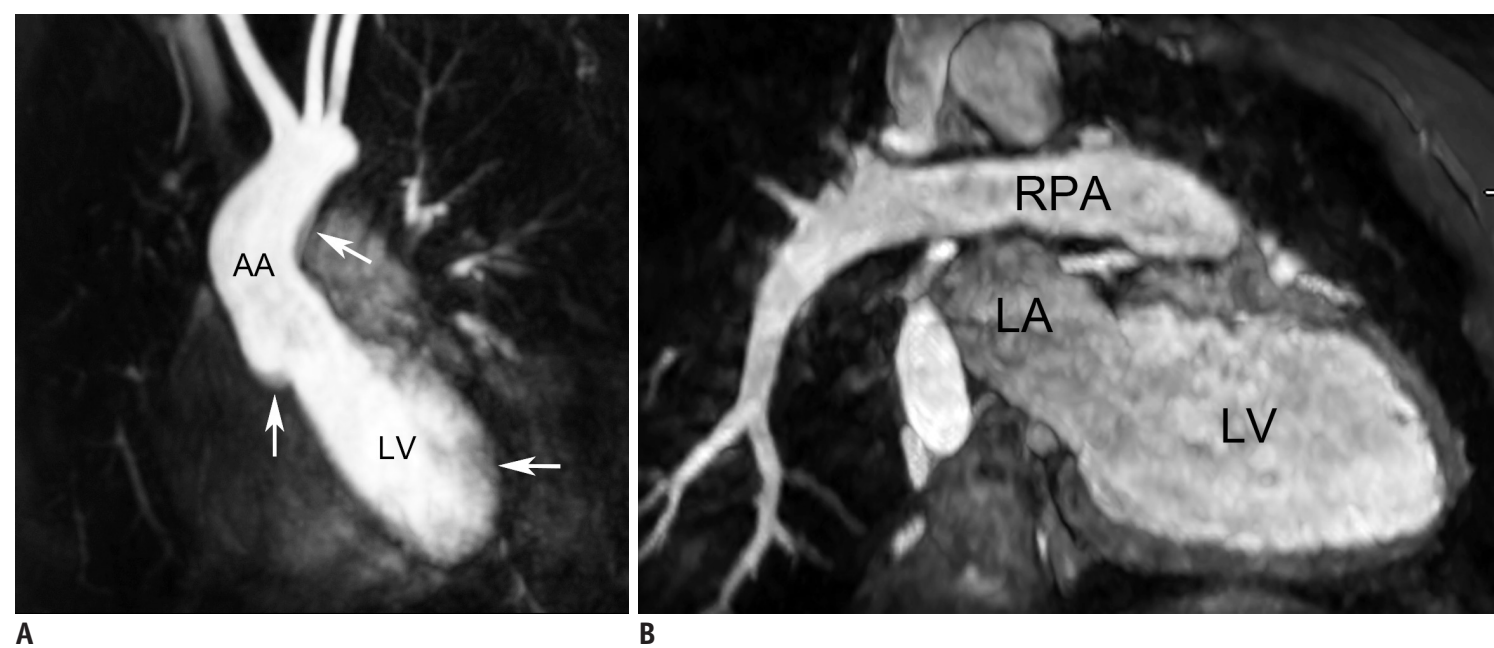

Fig. 1. 3D cardiac MRI.

A. Oblique coronal view of time-resolved non-ECG-synchronized contrast-enhanced 3D cardiac MRI showing motion artifacts (arrows) in AA and $\mathrm{LV}$, which is problematic in illustrating accurate cardiovascular morphology on advanced visualization techniques. B. Oblique coronal view of ECG-triggered, navigator-gated T2-prepared balanced steady-state free precession imaging demonstrating motionless cardiovascular morphology but suboptimal heterogeneous signal intensities in LA, LV, and RPA. AA = ascending aorta, ECG = electrocardiography, LA = left atrium, LV = left ventricle, $M R I=$ magnetic resonance imaging, $\mathrm{RPA}=$ right pulmonary artery, $3 \mathrm{D}=$ three-dimensional 
be illustrated (Fig. 2). Furthermore, 3D threshold-based segmentation is increasingly used for volume measurements of cardiac chambers for evaluating cardiac function in patients with CHD (13-15) (Fig. 3). A new cinematic rendering technique was recently introduced for 3D photorealistic visualization of complex cardiovascular structures by enhancing depth perception (16). Cinematic rendering may also be used to illustrate intracardiac structures by adjusting the opacity to make enhanced blood in cardiac chambers completely transparent (Fig. 4). A combined segmentation approach with deep-learning algorithms may accelerate cardiovascular post-processing (17).

\section{Reality-Virtual Continuum}

In augmented reality, virtual objects are overlaid on the real-world with spatial registration. In virtual reality, a user is immersed in an entirely artificial digital environment. Thus, augmented reality is clearly distinguished from virtual reality in terms of the presence of the ability to still visualize the real-world. On the other hand, the concept of mixed reality, defined as anywhere in the so-called "reality-virtual continuum," ranging from the completely real to a completely virtual environment, was proposed in 1994 (18). Compared to augmented reality, a mixed reality not only overlays but anchors virtual objects to real-world objects and allows

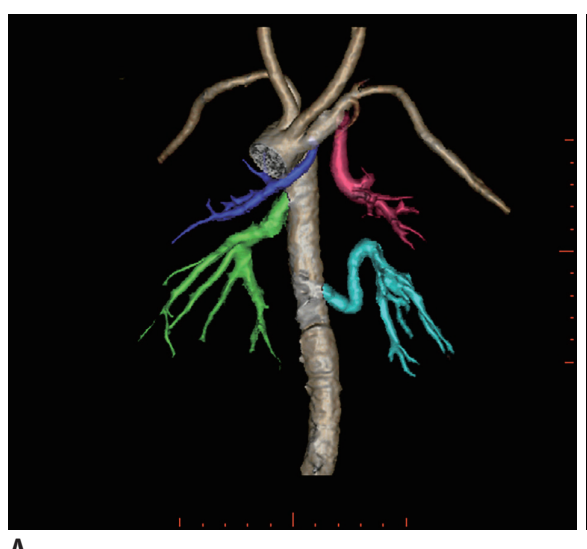

A

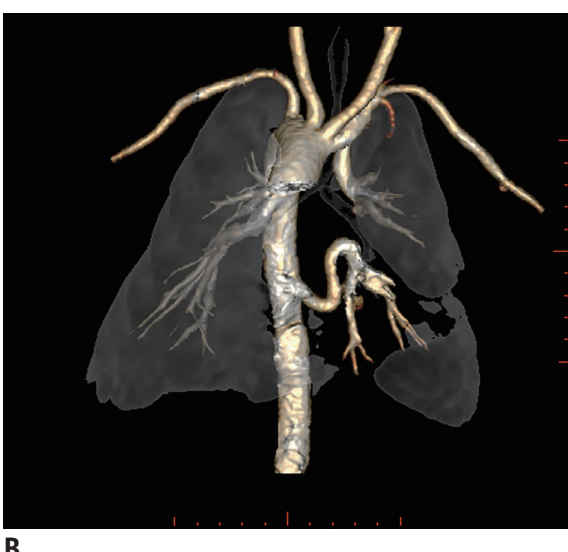

B

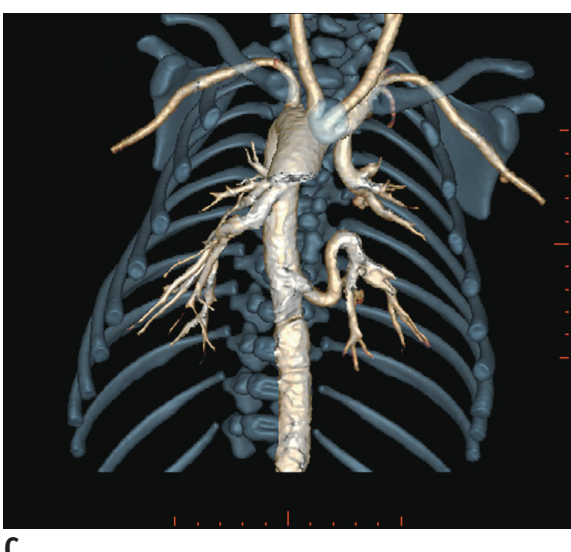

C

Fig. 2. Advanced post-processing of 3D cardiothoracic CT imaging.

A. In coronal volume-rendered CT images with major aortopulmonary collateral arteries, each artery can be illustrated in distinct color. In addition, other structures such as airways and lungs (B) and thoracic cage (C) may be rendered simultaneously to enhance their spatial relationships with collateral arteries, which is helpful for pre-procedural planning. CT = computed tomography

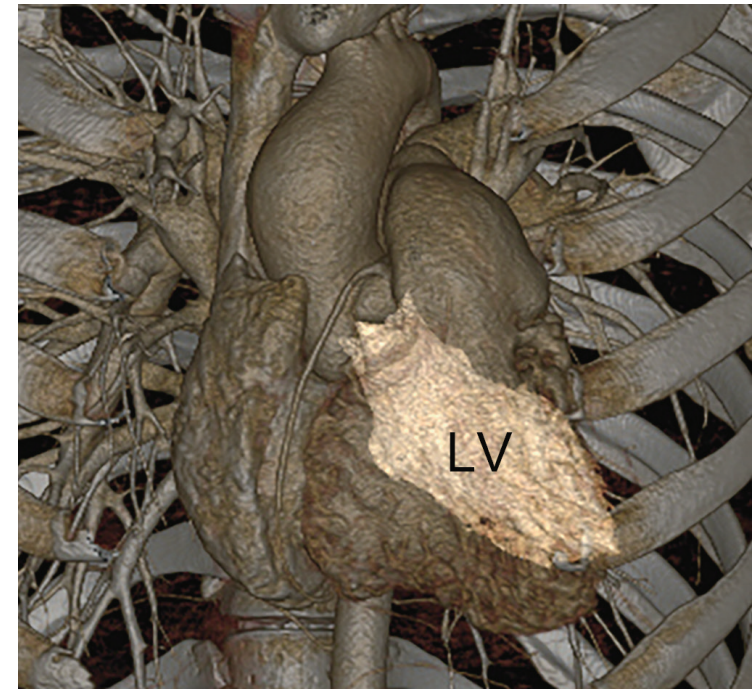

A

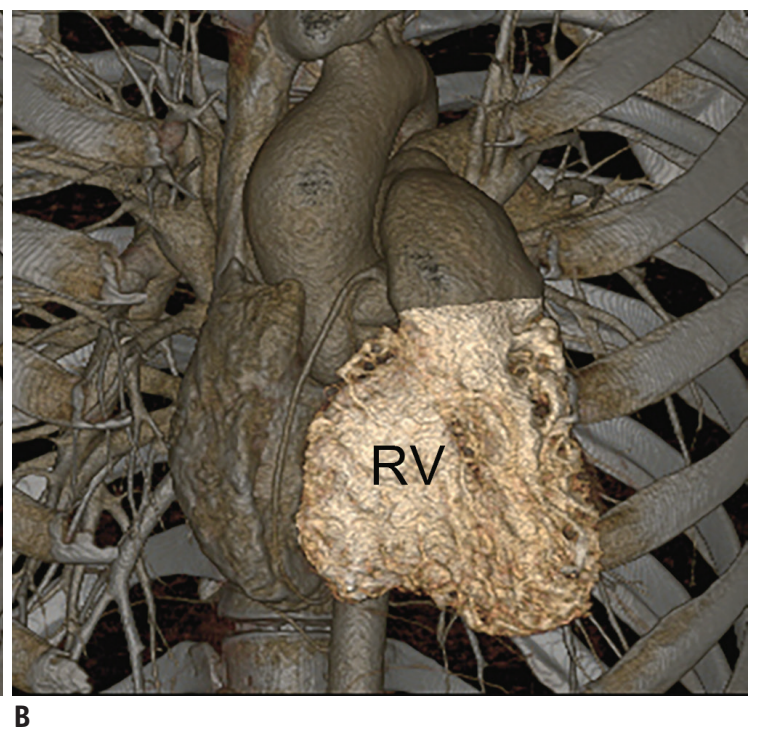

Fig. 3. CT ventricular volumetry using 3D threshold-based segmentation.

Coronal volume-rendered CT images highlight LV (A) and RV (B) segmented for ventricular volumetry using 3D threshold-based approach. $\mathrm{RV}=$ right ventricle 

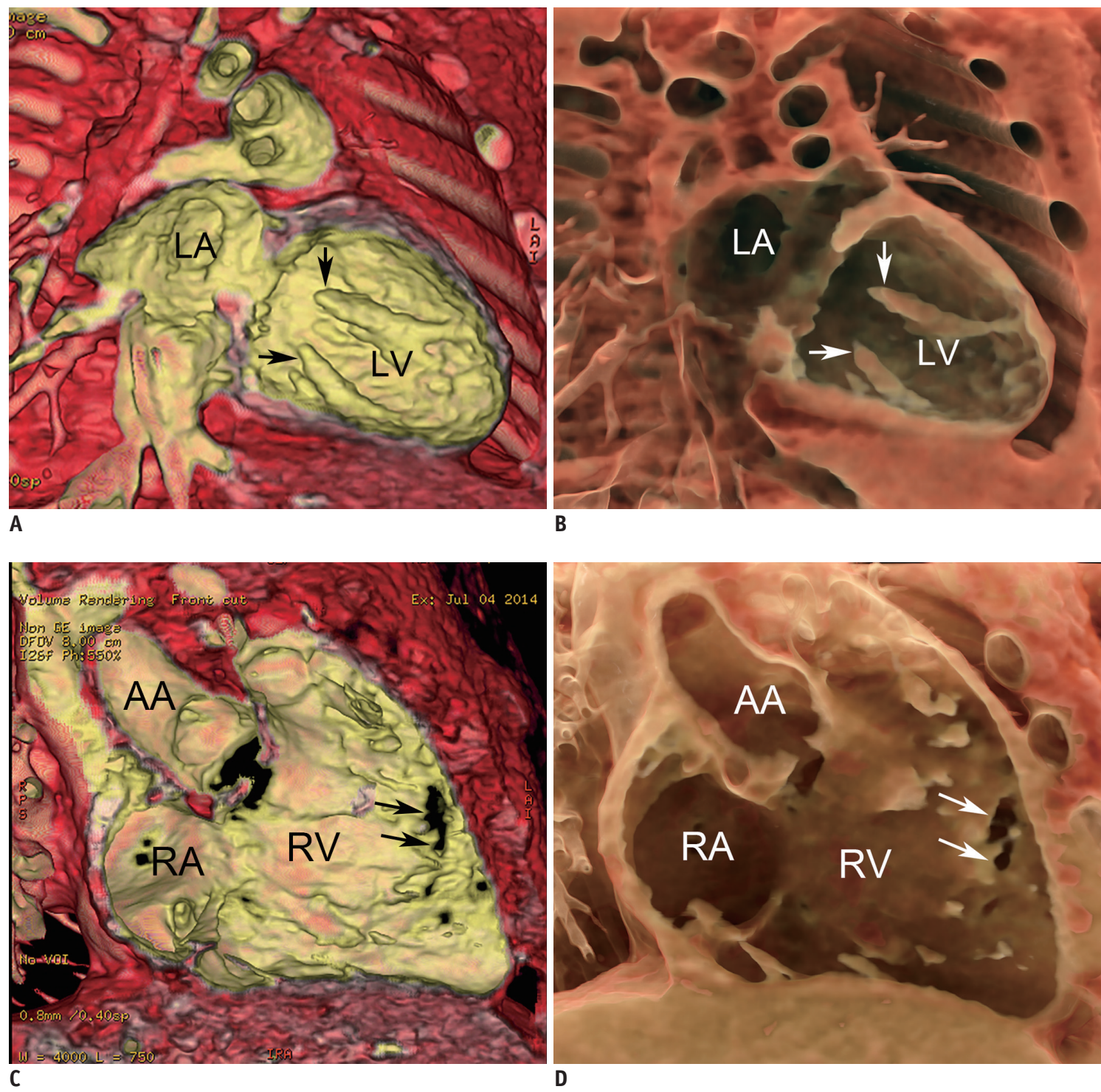

Fig. 4. Transparent-lumen cinematic rendering.

Depth perception of cinematic-rendered images (B, D) are superior to those on regular volume-rendered images $(\mathbf{A}, \mathbf{C})$. Therefore, papillary muscles (arrows; A, B) and trabeculations are better visualized on cinematic-rendered images (B, D). With improved depth perception, anterior marginal muscular VSDs (arrows; C, D) are demonstrated without additional back clipping that must be used for regular volume-rendered image (C). Realistic endocardial appearance of transparent-lumen cinematic rendering mimics 3D printed, hollow cardiac model. RA = right atrium, VSD = ventricular septal defect

the user to interact with combined virtual and real objects. Notably, segmented and processed medical images are universally used in all three of these advanced visualization techniques (Fig. 5). Clinically, virtual 3D cardiac modeling has recently become regarded as an invaluable alternative to cadaveric heart specimens that are difficult to obtain and subject to tissue degradation over time $(19,20)$. These 3D digital models can be viewed within different visualization methods and printed as a 3D physical replica. However, it should be noted that virtual 3D modeling requires laborintensive, time-consuming processing and may provide lower image quality than a typical volume rendering $(21,22)$.

\section{Augmented and Mixed Realities}

\section{Techniques}

Fundamental elements of augmented reality include real objects (such as patients), virtual objects processed from medical images, spatial registration, instrument tracking, and visualization. The combined visualization of spatially registered virtual and real objects in augmented reality allows clinicians to maintain focus and work 


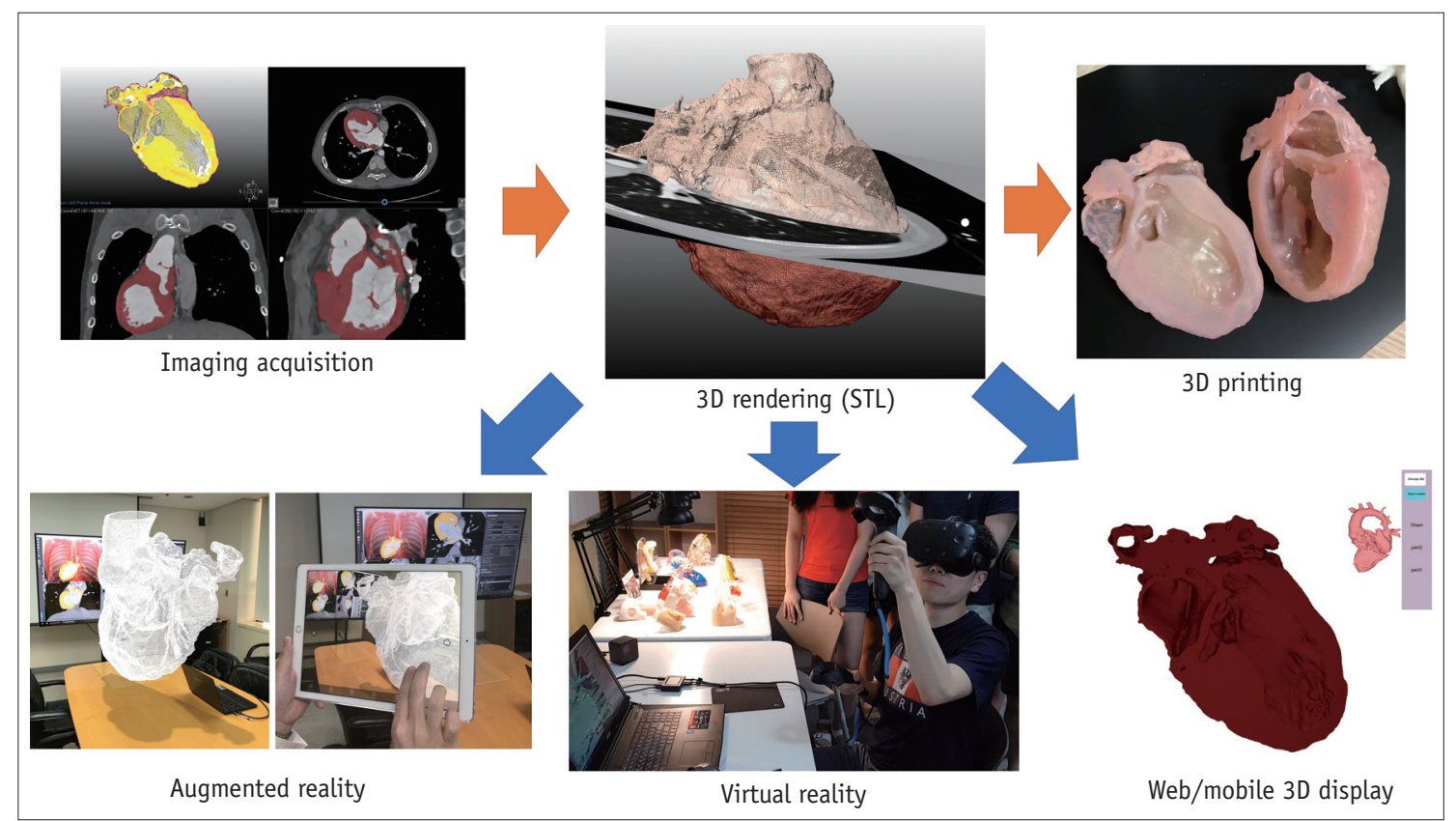

Fig. 5. Workflow of advanced visualization technology. Segmented and refined 3D model can be used not only for augmented reality, virtual reality, and interactive web or mobile 3D displays but also for 3D printing. STL = Standard Tessellation Language

without taking their eyes off their patients. Thus clinicians avoid distractions and can concentrate on the procedure, while maintaining their ability to detect unanticipated complications. Spatial registration has been mostly applied to rigid organs. Accurate registration is difficult to achieve in soft or moving organs due to extensive elastic deformation, which is why applying augmented reality to CHD has been extraordinarily cumbersome. A tracking device is necessary for surgical or interventional navigation. Currently, optical, or electromagnetic tracking systems are used, and each of these has its benefits and limitations $(1,23)$. In order to overlay virtual objects on real objects, a variety of display methods, such as conventional display monitors, headmounted displays, projection displays, and transparent screens, may be used. Visualization using augmented reality is insufficient for expressing depth perception. This shortcoming could be improved by seamlessly switching between augmented reality and virtual reality (24). The main barriers to the extensive use of augmented reality in medicine include the initial setup costs, additional time required to prepare virtual data, and unreliable accuracy in spatial registration. On October 24, 2018, the United States Food and Drug Administration cleared the first mixed reality medical solution, HoloLens ${ }^{\circledR}$ (Microsoft, Redmond, WA, USA), for use in pre-operative surgical planning. The technology can overlay two-dimensional, 3D, and four-dimensional images of patients onto the patient's body and provides true 3D depth perception. Holography, a 3D image formed by the interference of light beams from a laser or other coherent light source, recently became available for providing an intuitive and interactive real-time display of virtual objects in augmented or mixed reality without the need for any head-mounted devices by using a product called Holoscope ${ }^{\circledR}$ (RealView Imaging, Yokneam, Israel) (25).

\section{Applications}

In CHD, augmented reality can be used for education, preprocedural planning and simulation, and procedural guidance. Augmented reality is particularly helpful for minimally invasive cardiac intervention or surgery. It was demonstrated that augmented reality-enhanced transesophageal echocardiography could help to determine the optimal annuloplasty ring size (particularly for surgeons with limited experience), and for percutaneous mitral valve repair (26). Another study demonstrated that augmented reality using ECG-gated CT data facilitated transcatheter heart valve implantation during valve-in-valve procedures while using less contrast agent (27). In a patient with congenitally corrected transposition of the great arteries, augmented reality was used to guide transcatheter pacemaker implantation (28). Mixed reality using HoloLens ${ }^{\circledR}$ has helped surgeons understand the complex morphologies of CHD (29). 


\section{Virtual Reality}

\section{Techniques}

In virtual reality, 3D cardiac models should be generated first from medical images. Dynamic cardiac models may be produced from a static model using non-linear registration for virtual reality (30). For interactive visualization and manipulation of virtual objects, virtual reality equipmentsuch as headsets, controllers, and base stations to detect the spatial locations of the user and the controllersis necessary. The users may lose their sense of orientation when virtual objects are scaled up and rotated extensively. Furthermore, the entire virtual environment of virtual reality (completely separated from the real environment) is regarded as having narrow medical applicability due to patient safety concerns.

\section{Applications}

Until now, the medical use of virtual reality in the field of CHD has been quite limited. In 2001, virtual reality was first applied to interactive, real-time visualization of cardiac morphology (31). Its complementary role was recently demonstrated in two infants with CHD (32).

\section{Three-Dimensional Printing}

\section{Techniques}

Charles Hull introduced the first 3D printing technology in 1986. Since then, technical developments in medical 3D printing have evolved continually. The workflow for 3D printing comprises several sequential stages: 1) 3D data acquisition, 2) segmentation, 3) conversion of a Digital Imaging and Communication in Medicine (DICOM) file to a 3D mesh file format, 4) computer-aided design (CAD),
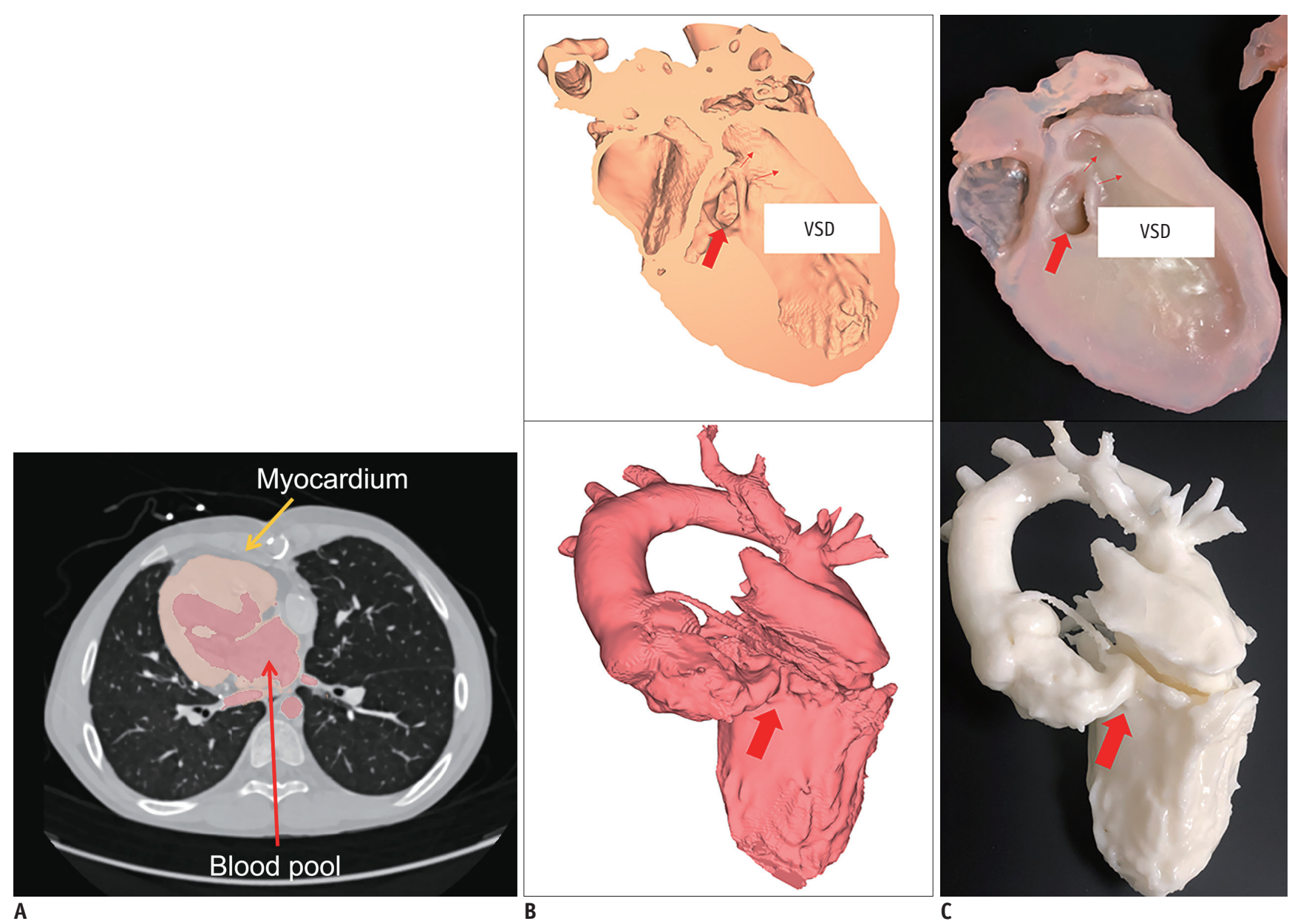

Fig. 6. 3D printing workflow tailored to congenital heart disease.

A. Axial CT image shows segmented blood pool mask and segmented myocardium mask with distinct colors. B. 3D virtual cardiac myocardial (upper) and blood pool (lower) models. C. 3D printed cardiac myocardial (upper) and blood pool (lower) models. Small VSD (large arrow) needs to be widened in direction of small arrows. 
and 5) 3D printing (Fig. 6). Acquisition of high-resolution isotropic 3D image data with excellent image quality is a mandatory prerequisite for creating a quality 3D printed model. In this regard, cardiac CT is the most commonly used 3D medical imaging modality for CHD 3D printing (2). Less frequently, 3D cardiac MRI (33), 3D echocardiography (34), or rotational angiography (35) have been used. Models used for CHD 3D printing such as a blood pool (a cast model) or wall (a hollow model with 1-2 mm minimum wall thickness) of cardiovascular structures must be segmented using dedicated software tools developed for generating Standard Tessellation Language (STL) files or various alternate file types such as object file $(\mathrm{OBJ})$, 3D manufacturing format (3MF), virtual reality modeling language (VRML). Mesh generation for the polygon structure is necessary to use CAD. There are several software programs ranging from expensive fully-featured commercial options such as Mimics (Materialise, Leuven, Belgium) and D2P (3D Systems, Rock Hill, SC, USA), midrange versions such as Medip (MEDICALIP, Seoul, Korea), and open-source freeware platforms such as Slicer (http:// www.slicer.org) and itk-SNAP (http://www.itksnap.org). Thresholding, region growing, and manual editing are used for segmentation. Exceptional expertise in the field of CHD is required for accurate and relevant segmentation. Even for top experts, segmentation is time-consuming (usually 2-3 hours) depending on the image quality of the original 3D medical imaging data $(2,3)$.

Consequently, the importance of the original data cannot be overemphasized for obtaining quality STL files and printed models as well as for reducing image processing time. Newer software tools using semiautomatic segmentation may reduce time and improve reproducibility (36), but further refinement (e.g., wrapping or smoothing) using CAD software is required because the segmented STL images are not optimized for $3 \mathrm{D}$ printing in most cases. The process of 'wrapping' creates a watertight 3D surface by filling any holes, and the 'smoothing' process is often needed to make the 3D surface regular and even. Moreover, CAD software can be used to add artificial tissue such as a ventricular septal defect patch to a patient-specific 3D cardiac model (37).

The refined STL files are loaded into a 3D printer, then printing is started with appropriately selected materials. A variety of $3 \mathrm{D}$ printing technologies are available in cardiovascular medicine, including fused deposition modeling, selective laser sintering, stereolithography, material jetting, and injection molding; the details have been already described elsewhere (4, 38-40). A wide range of printing materials may be selected, including plastic (resin), silicone, nylon, and metal, as needed, and are primarily divided into rigid vs. soft rubber-like materials, opaque vs. transparent, and single-color vs. multi-color (4, 38-43). However, soft materials are still quite different from real cardiovascular tissues. Therefore, surgeons find the models more difficult to suture because they are easily torn or cut $(2,44)$ (Fig. 7). A new flexible material (Agilus30 white, Stratasys, Rehovot, Israel), which is less prone to tearing, was recently introduced in November 2018, to compensate for these shortcomings. After a model is printed, a cleaning or finishing process is required, and sufficient time should be spent finishing the newly printed material. The time required to make the final product (as much as 24 hours), the cost of printing one unit (1000-3500 US dollars) as well as the quality of the printed models are quite different depending on the selected printer, printing material, model size, and complexity of cardiac morphology $(2,4,33,45-48)$. Recently, the time required for detailed

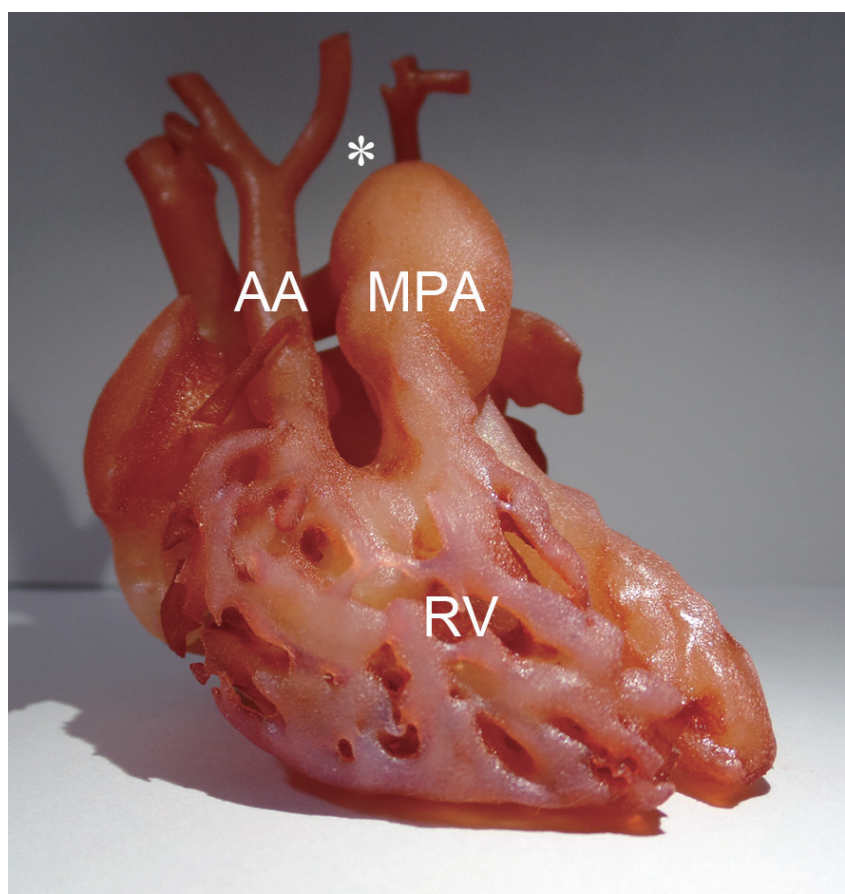

Fig. 7. Patient-specific 3D printed hollow cardiac model using flexible printing material (TangoPlus, Stratasys) from 3D cardiothoracic CT data obtained preoperatively in infant with double outlet RV and interrupted aortic arch type B. Both smaller AA and dilated MPA arise from double outlet RV. Aortic arch is interrupted (asterisk) between left common carotid artery and left subclavian artery, indicating type B of interrupted aortic arch. MPA = main pulmonary artery 
segmentation was markedly reduced by using direct conversion from a segmented DICOM file to an STL file, available in a few clinical high-performance 3D workstations for CT and MRI (Fig. 8).

\section{Applications}

Binder et al. (49) first applied 3D printing from echocardiographic data to cardiac structures in 2000. In the last two decades, 3D printing has increasingly been used for diagnosis, pre-procedural planning and simulation, and different levels of training and education for a variety of simple and complex CHDs $(2,32-34,37,45,50-52)$. It is not surprising that $3 \mathrm{D}$ printing accurately replicates $3 \mathrm{D}$ digital models and 3D source images to a submillimeter level when considering the mechanical precision of the technology $(32,33,52)$. Thus, most errors in $3 \mathrm{D}$ printing are introduced during segmentation or via CAD.

Further medical value of 3D printing for $\mathrm{CHD}$ has been evaluated predominantly through subjective assessments and questionnaires without correlation to outcome measures mostly in case series or small studies $(47,51,52)$, and even in a prospective multi-center study (30). The added value should be more meaningful and more evident in complex cardiac defects than in uncomplicated cardiac defects. The required quality of $3 \mathrm{D}$ printed cardiac models is not the same in all medical applications. For example, the quality of 3D printed cardiac models for hands-on surgical simulation should be sufficiently high to mimic genuine cardiovascular tissue as much as possible, while such high-quality models may not be necessary for education and communication. In urgent clinical cases, the time required for the whole 3D printing process should be strictly controlled so that the resulting device is completed promptly (e.g., within 2-3 business days).

CT or MRI may be used to create a 3D printed cardiac valve model if imaging is acquired when the cardiac valves show minimal motions (Fig. 9). Alternatively, artificial valves created using CAD may be added to a patientspecific 3D cardiac model (2). Because CT or MRI cannot provide high-quality images of delicate and fast-moving cardiac values, 3D echocardiography is used to create a 3D printed cardiac valve models (43). Hybrid 3D printing, which integrates the strengths of 3D echocardiography to define the cardiac valves and the strengths of CT or MRI to define other cardiovascular structures, has been attempted $(53,54)$. Patient-specific cardiovascular phantoms using 3D printing may be designed for device testing or advanced hemodynamic evaluation (55).

\section{Unresolved Issues}

We need a variety of expert opinions involved to advance 3D visualization techniques of cardiovascular structures in CHD. We first compare these techniques in various aspects (Table 1). Several studies have tackled problems of $3 \mathrm{D}$ printing in terms of cost, time, and changeability by comparing it with virtual 3D modeling. For example, a bedside display of virtual 3D cardiac models using a tablet device was as effective as more expensive 3D printed models in a cardiac intensive care unit (50). Other limitations of 3D printed cardiac models are a lack of dynamic representations throughout the entire cardiac cycle and

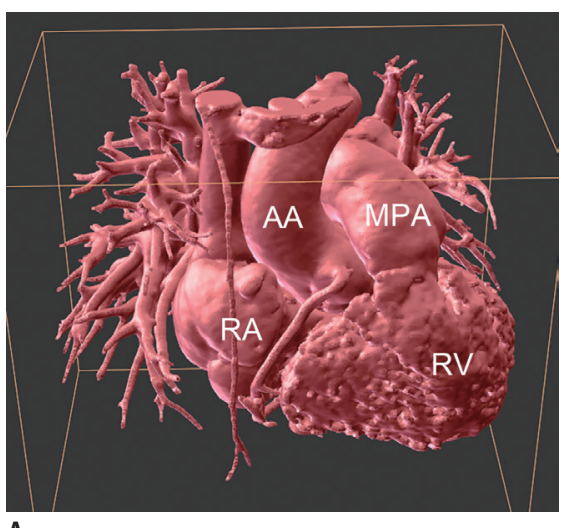

A
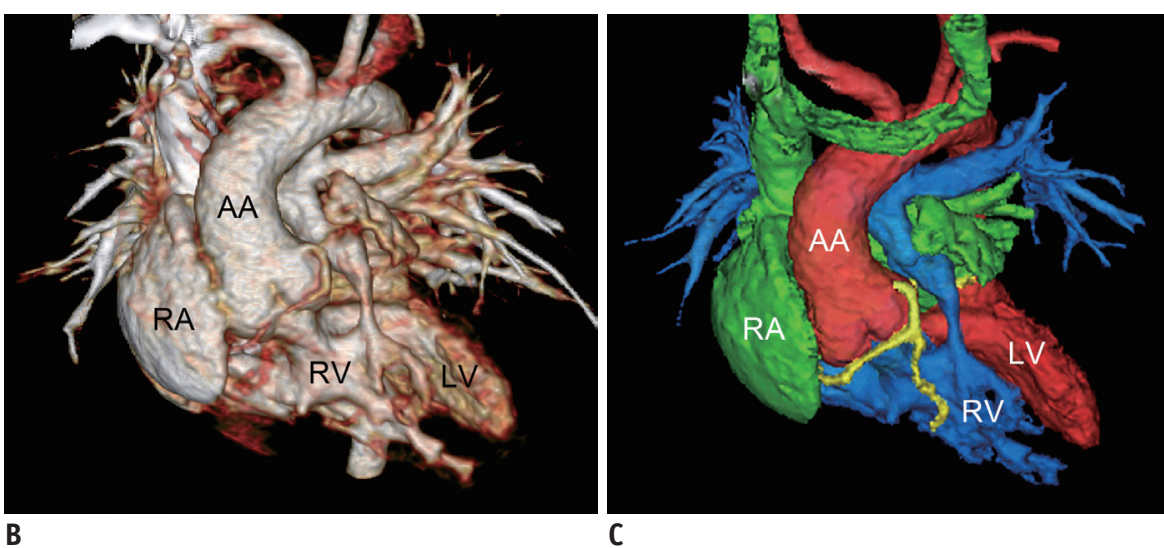

Fig. 8. Direct file conversion from segmented DICOM file to STL file. Segmented cardiovascular volume in DICOM format can be directly converted to STL format.

Segmented part may be single volume (A, B) or merged volume comprising multiple parts (C). DICOM = Digital Imaging and Communication in Medicine 


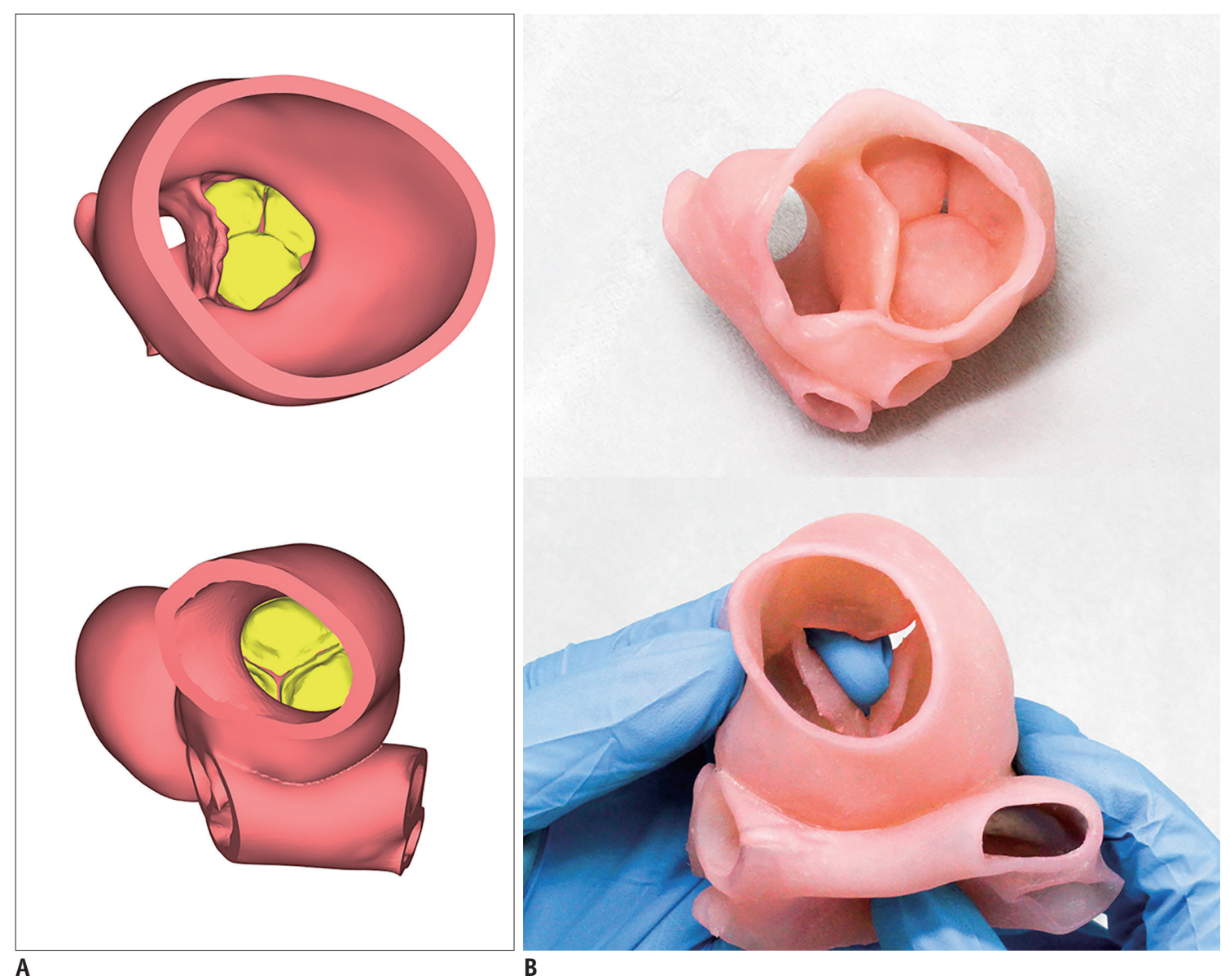

Fig. 9. 3D printed aortic valve model for surgical simulation.

A. Aortic valve (yellow) is seen in exported polygon rendered STL file. B. 3D printed model is used for surgical simulation of aortic valve. Surgeon may open aortic valve with their finger.

Table 1. Comparison between Advanced 3D Visualization Techniques in Congenital Heart Disease

\begin{tabular}{|c|c|c|c|c|c|c|c|}
\hline $\begin{array}{c}\text { 3D } \\
\text { Visualization Techniques }\end{array}$ & $\begin{array}{l}\text { Full Voxel } \\
\text { Information }\end{array}$ & $\begin{array}{c}\text { Depth } \\
\text { Perception }\end{array}$ & Changeability & CAD & Tangibility & $\begin{array}{c}\text { Additional Cost } \\
\text { for Hardware and } \\
\text { Software }\end{array}$ & $\begin{array}{c}\text { Additional Cost for } \\
\text { Printing Material and } \\
\text { Additional Printing Time }\end{array}$ \\
\hline $\begin{array}{l}\text { Standard 3D rendering } \\
\text { (DICOM format) }\end{array}$ & + & \pm & + & - & - & - & - \\
\hline Augmented/mixed reality & \pm & \pm & + & + & \pm & + & - \\
\hline Virtual reality & - & + & + & + & \pm & + & - \\
\hline 3D virtual model (STL format) & - & - & + & + & - & + & - \\
\hline $3 \mathrm{D}$ printed model & - & + & - & + & + & + & + \\
\hline
\end{tabular}

$\mathrm{CAD}=$ computer-aided design, DICOM = Digital Imaging and Communication in Medicine, STL = Standard Tessellation Language, 3D = three-dimensional

making use of hemodynamic data $(46,54)$. Both are crucial for understanding the pathophysiology of CHD.

Regarding cardiac phases in 3D imaging data, several studies suggested that the end-diastolic phase is preferred to the end-systolic phase for $3 \mathrm{D}$ printing because the end-diastolic phase shows a greater cardiac chamber size and more closely resembles the arrested heart during operation $(33,46)$. However, clinically relevant dimensions of ventricular outflow tracts and great arteries should be evaluated at the end-systolic phase (46). In other words, clinically significant outflow tract obstruction or great arterial dilatation can be overlooked in hands-on surgical simulation using 3D cardiac printed models acquired the end-diastolic phase. Furthermore, we should keep in mind 
that the end-systolic acquisition allows the best image quality at high heart rates typical in young children.

When we consider cost-benefit analysis, simple diagnostic tasks should be evaluated using already existing tools. The best strategy is the easiest solution. Superfluous use of augmented, mixed, or virtual realities and 3D printing, is attributable to the availability of $3 \mathrm{D}$ medical imaging reconstruction tools that are currently restricted to radiology departments in many institutions. In this regard, handson training could be provided to pediatric cardiac surgeons and pediatric cardiologists using a 3D medical imaging reconstruction tool on an inexpensive computer workstation (such as a common thin-client).

As in other new medical technologies used for patients, 3D printing needs standardization in terms of accuracy and reproducibility, guidelines for appropriateness criteria, and quality control by health authorities (38). Medical reimbursement is another practical obstacle for widespread clinical use of 3D printing $(38,41,56,57)$. There needs to be quantitative proof of improved patient outcomes and cost savings from the use of medical cardiac 3D printing to expedite the reimbursement process. In the United States, the medical use of 3D printing was assigned to a Current Procedural Terminology Category III in 2018, which makes it only voluntarily reimbursable. In South Korea, 3D printing was approved as a New Health Technology specifically for surgical simulation of CHD in December 2018.

The establishment of a national multidisciplinary 3D printing consortium sharing resources may be helpful to maximize the cost-effectiveness of many small demands on our current systems. We may disapprove if others use their 3D source data or segmented images without permission. Therefore, it is imperative to develop regulations protecting intellectual property in 3D printing.

Along with increased use of $3 \mathrm{D}$ printing, environmental issues related to 3D printing should be scrutinized. Ultrafine particles and volatile organic compounds emitted during the $3 \mathrm{D}$ printing process may cause occupational health issues, such as an increased risk of respiratory disease and cancer (58-60). Another environmental issue stems from the fact that $3 \mathrm{D}$ printing materials cannot be recycled in most cases $(22,58)$.

\section{FUTURE PERSPECTIVES}

User-friendliness, flexibility, portability, and costbenefit analyses will determine the future of augmented, mixed, and virtual reality for evaluating CHD. Those technologies will continue to compete with existing, proven 3D visualization techniques, chiefly utilized in radiology, and with 3D printing.

Hands-on surgical training using 3D cardiac printed models in CHD will contribute to surgeons' skill development. A hands-on course may be recognized as a standard component of congenital heart surgical training programs and board certification processes with further validation of its impact on clinical outcomes. A digital library of 3D images and 3D printed models embracing the entire spectrum of CHD should be established and would be tremendously beneficial for medical education.

Within the scope of personalized medicine, patientspecific implants using 3D printing technology will be more widely used for patients with CHD and will contribute to improved clinical outcomes. Hemodynamic assessment in living individuals is usually an arduous task because of many confounding factors. Graphically designed healthy and pathological models may aid hypothesis testing because confounding factors can be eliminated in experiments. Additionally, hemodynamics of the simulated blood flow circuits can be assessed using Doppler ultrasound or phasecontrast MRI by connecting 3D printed models to flow pumps.

Bioprinting technologies in 3D are advancing slowly, and they are still predominantly used pre-clinically $(61,62)$. An example of 3D bioprinting is the printing of tissue or an organ with so-called "bioink" composed of live cells, usually with an additional carrier material that envelops the cells. Most frequently, the carrier material is a biopolymer gel that acts as a 3D scaffold for cells to attach, spread, grow, and proliferate. The potential applications of 3D bioprinting in CHD include the production of autografts for vascular grafts, valve replacement, and heart transplantation.

\section{Conflicts of Interest}

The authors have no potential conflicts of interest to disclose.

\section{ORCID ID}

Hyun Woo Goo

https://orcid.org/0000-0001-6861-5958

\section{REFERENCES}

1. Hong J. Medical augmented reality and virtual reality. J Korean Soc Radiol 2019;80:226-238 
Augmented Reality, Mixed Reality, Virtual Reality and Three-Dimensional Printing

2. Yoo SJ, Thabit 0, Kim EK, Ide H, Yim D, Dragulescu A, et al. $3 \mathrm{D}$ printing in medicine of congenital heart diseases. 3D Print Med 2015;2:3

3. Kim GB, Lee S, Kim H, Yang DH, Kim YH, Kyung YS, et al. Three-dimensional printing: basic principles and applications in medicine and radiology. Korean J Radiol 2016;17:182-197

4. Byrne N, Velasco Forte M, Tandon A, Valverde I, Hussain T. A systematic review of image segmentation methodology, used in the additive manufacture of patient-specific 3D printed models of the cardiovascular system. JRSM Cardiovasc Dis 2016;5:2048004016645467

5. Goo HW. State-of-the-art CT imaging techniques for congenital heart disease. Korean J Radiol 2010;11:4-18

6. Goo HW. Current trends in cardiac CT in children. Acta Radiol 2013;54:1055-1062

7. Goo HW. CT radiation dose optimization and estimation: an update for radiologists. Korean J Radiol 2012;13:1-11

8. Hui PKT, Goo HW, Du J, Ip JJK, Kanzaki S, Kim YJ, et al. Asian consortium on radiation dose of pediatric cardiac CT (ASCIREDCARD). Pediatr Radiol 2017;47:899-910

9. Hong SH, Goo HW, Maeda E, Choo KS, Tsai IC; Asian Society of Cardiovascular Imaging Congenital Heart Disease Study Group. User-rriendly vendor-specific guideline for pediatric cardiothoracic computed tomography provided by the Asian Society of Cardiovascular Imaging Congenital Heart Disease Study Group: part 1. Imaging techniques. Korean J Radiol 2019;20:190-204

10. Fratz S, Chung T, Greil GF, Samyn MM, Taylor AM, Valsangiacomo Buechel ER, et al. Guidelines and protocols for cardiovascular magnetic resonance in children and adults with congenital heart disease: SCMR expert consensus group on congenital heart disease. J Cardiovasc Magn Reson 2013;15:51

11. Makowski MR, Wiethoff AJ, Uribe S, Parish V, Botnar RM, Bell $A$, et al. Congenital heart disease: cardiovascular MR imaging by using an intravascular blood pool contrast agent. Radiology 2011;260:680-688

12. Zhou Z, Han F, Rapacchi S, Nguyen KL, Brunengraber DZ, Kim GJ, et al. Accelerated ferumoxytol-enhanced 4D multiphase, steady-state imaging with contrast enhancement (MUSIC) cardiovascular MRI: validation in pediatric congenital heart disease. NMR Biomed 2017;30:e3663

13. Goo HW. Comparison between three-dimensional navigatorgated whole-heart MRI and two-dimensional cine MRI in quantifying ventricular volumes. Korean J Radiol 2018;19:704714

14. Goo HW. Semiautomatic three-dimensional threshold-based cardiac computed tomography ventricular volumetry in repaired tetralogy of Fallot: comparison with cardiac magnetic resonance imaging. Korean J Radiol 2019;20:102-113

15. Goo HW. Volumetric severity assessment of Ebstein anomaly using three-dimensional cardiac CT: a feasibility study. Cardiovasc Imaging Asia 2019;3:61-67

16. Rowe SP, Johnson PT, Fishman EK. Cinematic rendering of cardiac CT volumetric data: principles and initial observations.
J Cardiovasc Comput Tomogr 2018;12:56-59

17. Avendi MR, Kheradvar A, Jafarkhani H. A combined deeplearning and deformable-model approach to fully automatic segmentation of the left ventricle in cardiac MRI. Med Image Anal 2016;30:108-119

18. Milgram P, Kishino F. A taxonomy of mixed reality visual displays. IEICE Trans Inf Syst 1994;E77-D:1321-1329

19. Seslar SP, Shepard CW, Giroud JM, Aiello VD, Cook AC, Spicer $D E$, et al.; Archiving Working Group of The International Society for Nomenclature of Paediatric and Congenital Heart Disease. Lost treasures: a plea for the systematic preservation of cadaveric heart specimens through three-dimensional digital imaging. Cardiol Young 2015;25:1457-1459

20. Kiraly L, Kiraly B, Szigeti K, Tamas CZ, Daranyi S. Virtual museum of congenital heart defects: digitization and establishment of a database for cardiac specimens. Quant Imaging Med Surg 2019;9:115-126

21. Farooqi KM, Uppu SC, Nguyen K, Srivastava S, Ko HH, Choueiter $\mathrm{N}$, et al. Application of virtual three-dimensional models for simultaneous visualization of intracardiac anatomic relationships in double outlet right ventricle. Pediatr Cardiol 2016;37:90-98

22. Speggiorin S, Durairaj S, Mimic B, Corno AF. Virtual 3D modeling of airways in congenital heart defects. Front Pediatr 2016;4:116

23. Peters TM, Linte CA. Image-guided interventions and computer-integrated therapy: quo vadis? Med Image Anal 2016;33:56-63

24. Choi H, Cho B, Masamune K, Hashizume M, Hong J. An effective visualization technique for depth perception in augmented reality-based surgical navigation. Int J Med Robot 2016;12:62-72

25. Bruckheimer E, Rotschild C, Dagan T, Amir G, Kaufman A, Gelman S, et al. Computer-generated real-time digital holography: first time use in clinical medical imaging. Eur Heart J Cardiovasc Imaging 2016;17:845-849

26. Ender J, Koncar-Zeh J, Mukherjee C, Jacobs S, Borger MA, Viola $C$, et al. Value of augmented reality-enhanced transesophageal echocardiography (TEE) for determining optimal annuloplasty ring size during mitral valve repair. Ann Thorac Surg 2008;86:1473-1478

27. Belhaj Soulami R, Verhoye JP, Nguyen Duc H, Castro M, Auffret $V$, Anselmi A, et al. Computer-assisted transcatheter heart valve implantation in valve-in-valve procedures. Innovations (Phila) 2016;11:193-200

28. Opolski MP, Michałowska IM, Borucki BA, Nicińska B, Szumowski $\measuredangle$, Sterliński M. Augmented-reality computed tomography-guided transcatheter pacemaker implantation in dextrocardia and congenitally corrected transposition of great arteries. Cardiol J 2018;25:412-413

29. Brun H, Bugge RAB, Suther LKR, Birkeland S, Kumar R, Pelanis $E$, et al. Mixed reality holograms for heart surgery planning: first user experience in congenital heart disease. Eur Heart $\mathrm{J}$ Cardiovasc Imaging 2019;20:883-888 
30. Wierzbicki M, Drangova M, Guiraudon G, Peters T. Validation of dynamic heart models obtained using non-linear registration for virtual reality training, planning, and guidance of minimally invasive cardiac surgeries. Med Image Anal 2004;8:387-401

31. Sørensen TS, Therkildsen SV, Makowski P, Knudsen JL, Pedersen EM. A new virtual reality approach for planning of cardiac interventions. Artif Intell Med 2001;22:193-214

32. Ong CS, Krishnan A, Huang CY, Spevak P, Vricella L, Hibino $\mathrm{N}$, et al. Role of virtual reality in congenital heart disease. Congenit Heart Dis 2018;13:357-361

33. Valverde I, Gomez-Ciriza G, Hussain T, Suarez-Mejias C, Velasco-Forte MN, Byrne N, et al. Three-dimensional printed models for surgical planning of complex congenital heart defects: an international multicentre study. Eur J Cardiothorac Surg 2017;52:1139-1148

34. Olivieri LJ, Krieger A, Loke YH, Nath DS, Kim PC, Sable CA. Three-dimensional printing of intracardiac defects from threedimensional echocardiographic images: feasibility and relative accuracy. J Am Soc Echocardiogr 2015;28:392-397

35. Parimi M, Buelter J, Thanugundla V, Condoor S, Parkar $\mathrm{N}$, Danon S, et al. Feasibility and validity of printing 3D heart models from rotational angiography. Pediatr Cardiol 2018;39:653-658

36. Tandon A, Byrne N, Nieves Velasco Forte Mde L, Zhang S, Dyer AK, Dillenbeck JM, et al. Use of a semi-automated cardiac segmentation tool improves reproducibility and speed of segmentation of contaminated right heart magnetic resonance angiography. Int J Cardiovasc Imaging 2016;32:1273-1279

37. Giannopoulos AA, Chepelev L, Sheikh A, Wang A, Dang W, Akyuz $E$, et al. 3D printed ventricular septal defect patch: a primer for the 2015 Radiological Society of North America (RSNA) hands-on course in 3D printing. 3D Print Med 2015;1:3

38. Giannopoulos AA, Mitsouras D, Yoo SJ, Liu PP, Chatzizisis YS, Rybicki FJ. Applications of 3D printing in cardiovascular diseases. Nat Rev Cardiol 2016;13:701-718

39. Anwar S, Singh GK, Miller J, Sharma M, Manning P, Billadello $\mathrm{JJ}$, et al. $3 \mathrm{D}$ printing is a transformative technology in congenital heart disease. JACC Basic Transl Sci 2018;3:294-312

40. Townsend K, Pietila T. 3D printing and modeling of congenital heart defects: a technical review. Birth Defects Res 2018;110:1091-1097

41. Shin J, Truong QA. Manufacturing better outcomes in cardiovascular intervention: 3D printing in clinical practice today. Curr Treat Options Cardiovasc Med 2018;20:95

42. Hoashi T, Ichikawa $H$, Nakata T, Shimada M, Ozawa $H$, Higashida A, et al. Utility of a super-flexible threedimensional printed heart model in congenital heart surgery. Interact Cardiovasc Thorac Surg 2018;27:749-755

43. Scanlan AB, Nguyen AV, Ilina A, Lasso A, Cripe L, Jegatheeswaran $A$, et al. Comparison of 3D echocardiogramderived $3 \mathrm{D}$ printed valve models to molded models for simulated repair of pediatric atrioventricular valves. Pediatr
Cardiol 2018;39:538-547

44. Vukicevic M, Mosadegh B, Min JK, Little SH. Cardiac 3D printing and its future directions. JACC Cardiovasc Imaging 2017;10:171-184

45. Biglino G, Capelli C, Wray J, Schievano S, Leaver LK, Khambadkone $\mathrm{S}$, et al. 3D-manufactured patient-specific models of congenital heart defects for communication in clinical practice: feasibility and acceptability. BMJ Open 2015;5:e007165

46. Cantinotti M, Valverde I, Kutty S. Three-dimensional printed models in congenital heart disease. Int J Cardiovasc Imaging 2017;33:137-144

47. Lau I, Sun Z. Three-dimensional printing in congenital heart disease: a systematic review. J Med Radiat Sci 2018;65:226236

48. Lau I, Wong YH, Yeong CH, Abdul Aziz YF, Md Sari NA, Hashim $S A$, et al. Quantitative and qualitative comparison of low- and high-cost 3D-printed heart models. Quant Imaging Med Surg 2019;9:107-114

49. Binder TM, Moertl D, Mundigler G, Rehak G, Franke M, Delle-Karth $G$, et al. Stereolithographic biomodeling to create tangible hard copies of cardiac structures from echocardiographic data: in vitro and in vivo validation. J Am Coll Cardiol 2000;35:230-237

50. Olivieri LJ, Zurakowski D, Ramakrishnan K, Su L, Alfares FA, Irwin MR, et al. Novel, 3D display of heart models in the postoperative care setting improves CICU caregiver confidence. World J Pediatr Congenit Heart Surg 2018;9:206213

51. Costello JP, Olivieri LJ, Su L, Krieger A, Alfares F, Thabit 0 , et al. Incorporating three-dimensional printing into a simulation-based congenital heart disease and critical care training curriculum for resident physicians. Congenit Heart Dis 2015;10:185-190

52. Olivieri LJ, Su L, Hynes CF, Krieger A, Alfares FA, Ramakrishnan K, et al. "Just-In-Time" simulation training using 3-D printed cardiac models after congenital cardiac surgery. World J Pediatr Congenit Heart Surg 2016;7:164-168

53. Kurup HK, Samuel BP, Vettukattil JJ. Hybrid 3D printing: a game-changer in personalized cardiac medicine? Expert Rev Cardiovasc Ther 2015;13:1281-1284

54. Gosnell J, Pietila T, Samuel BP, Kurup HK, Haw MP, Vettukattil $\mathrm{JJ}$. Integration of computed tomography and threedimensional echocardiography for hybrid three-dimensional printing in congenital heart disease. J Digit Imaging 2016;29:665-669

55. Biglino G, Verschueren P, Zegels R, Taylor AM, Schievano S. Rapid prototyping compliant arterial phantoms for in-vitro studies and device testing. J Cardiovasc Magn Reson 2013;15:2

56. Bramlet M, Olivieri L, Farooqi K, Ripley B, Coakley M. Impact of three-dimensional printing on the study and treatment of congenital heart disease. Circ Res 2017;120:904-907

57. Moore RA, Riggs KW, Kourtidou S, Schneider K, Szugye N, Troja $W$, et al. Three-dimensional printing and virtual surgery 
Augmented Reality, Mixed Reality, Virtual Reality and Three-Dimensional Printing

for congenital heart procedural planning. Birth Defects Res 2018;110:1082-1090

58. Short DB, Sirinterlikci A, Badger P, Artieri B. Environmental, health, and safety issues in rapid prototyping. Rapid Prototyp J 2015;21:105-110

59. Randolph SA. 3D printing: what are the hazards? Workplace Health Saf 2018;66:164

60. Chan FL, House R, Kudla I, Lipszyc JC, Rajaram N, Tarlo SM.
Health survey of employees regularly using 3D printers. Occup Med (Lond) 2018;68:211-214

61. Melchiorri AJ, Hibino N, Best CA, Yi T, Lee YU, Kraynak CA, et al. 3D-printed biodegradable polymeric vascular grafts. Adv Healthc Mater 2016;5:319-325

62. Sun Y, Zhang X, Li W, Di Y, Xing Q, Cao Q. 3D printing and biocompatibility study of a new biodegradable occluder for cardiac defect. J Cardiol 2019;74:182-188 